\title{
The left atrial appendage amputation during off-pump coronary artery bypass grafting prevents stroke
}

\author{
Daisuke Endo ${ }^{1}$, Tohru Asai ${ }^{1}$, Taira Yamamoto ${ }^{2}$, Atsumi Oishi ${ }^{3}$, Yasutaka Yokoyama ${ }^{4}$, Akie \\ Shimada $^{2}$, Kan Kajimoto ${ }^{3}$, Satoshi Matsushita ${ }^{1}$, Shizuyuki Dohi ${ }^{1}$, and Atsushi Amano ${ }^{1}$ \\ ${ }^{1}$ Juntendo University \\ ${ }^{2}$ Juntendo University Nerima Hospital \\ ${ }^{3}$ Juntendo Shizuoka Hospital \\ ${ }^{4}$ Toda Chuo General Hospital
}

June 14, 2021

\begin{abstract}
Background: Postoperative atrial fibrillation (POAF) occurs in $20 \%$ to $50 \%$ of patients soon after cardiac surgery, and is associated with subsequent newly developed AF. Left atrial appendage closure is effective in preventing stroke among patients with nonvalvular AF. However, the prophylactic effect of left atrial appendage amputation (LAAA) in stroke prevention among patients with sinus rhythm has not been proven. Methods: We analyzed 977 consecutive patients undergoing off-pump coronary artery bypass grafting (OPCAB) with sinus rhythm, with or without concomitant LAAA, from 2011 to 2017 at our institution in a retrospective observational manner. A total of 550 patients $(56.3 \%)$ underwent concomitant LAAA, any effect thereof in preventing early ( $<30$ days) and later stroke was examined. Results: The incidences of POAF and early stroke were not significantly different between the groups. The incidence of later stroke was higher in patients without LAAA than in those with LAAA $(4.0 \%$ vs. $1.6 \% ; \mathrm{p}=0.02)$. In a subanalysis of patients without LAAA, stroke occurred more frequently in those with POAF than those without POAF ( $7.5 \%$ vs. $2.7 \%$; $=0.03)$, while in patients receiving LAAA, stroke incidences did not differ between those with and without POAF. Multivariate cox proportional hazard model showed POAF without LAAA was the only independent predictor of stroke (HR: 2.86; 95\% CI: 1.10 to $7.45 ; \mathrm{p}=0.03$ ). Conclusions: Concomitant surgical LAA amputation during OPCAB reduces the risk of stroke.
\end{abstract}

\section{Hosted file}

LAAA manuscript for J Card Surg. 6.6.doc available at https://authorea.com/users/388743/ articles/526103-the-left-atrial-appendage-amputation-during-off-pump-coronary-arterybypass-grafting-prevents-stroke 

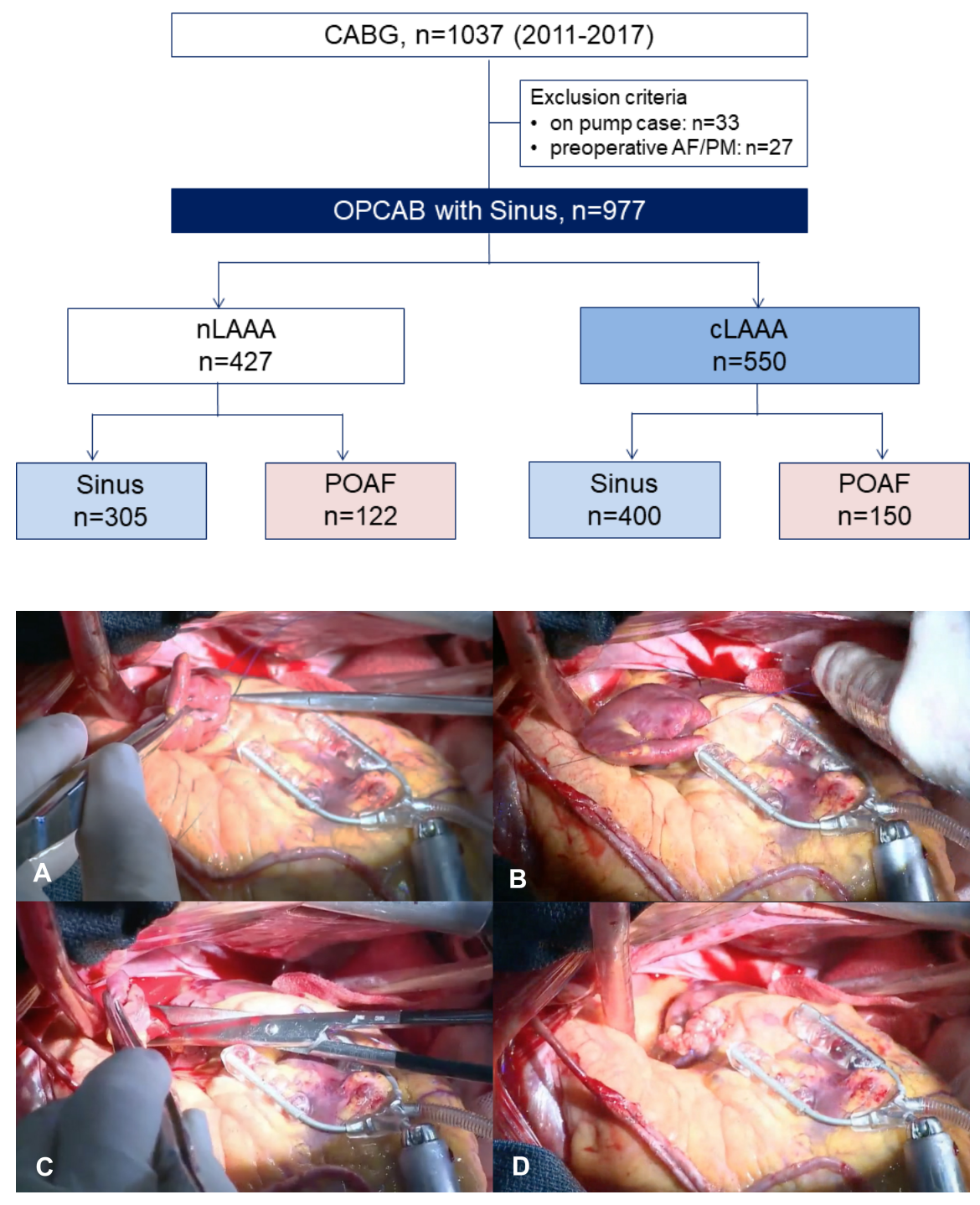

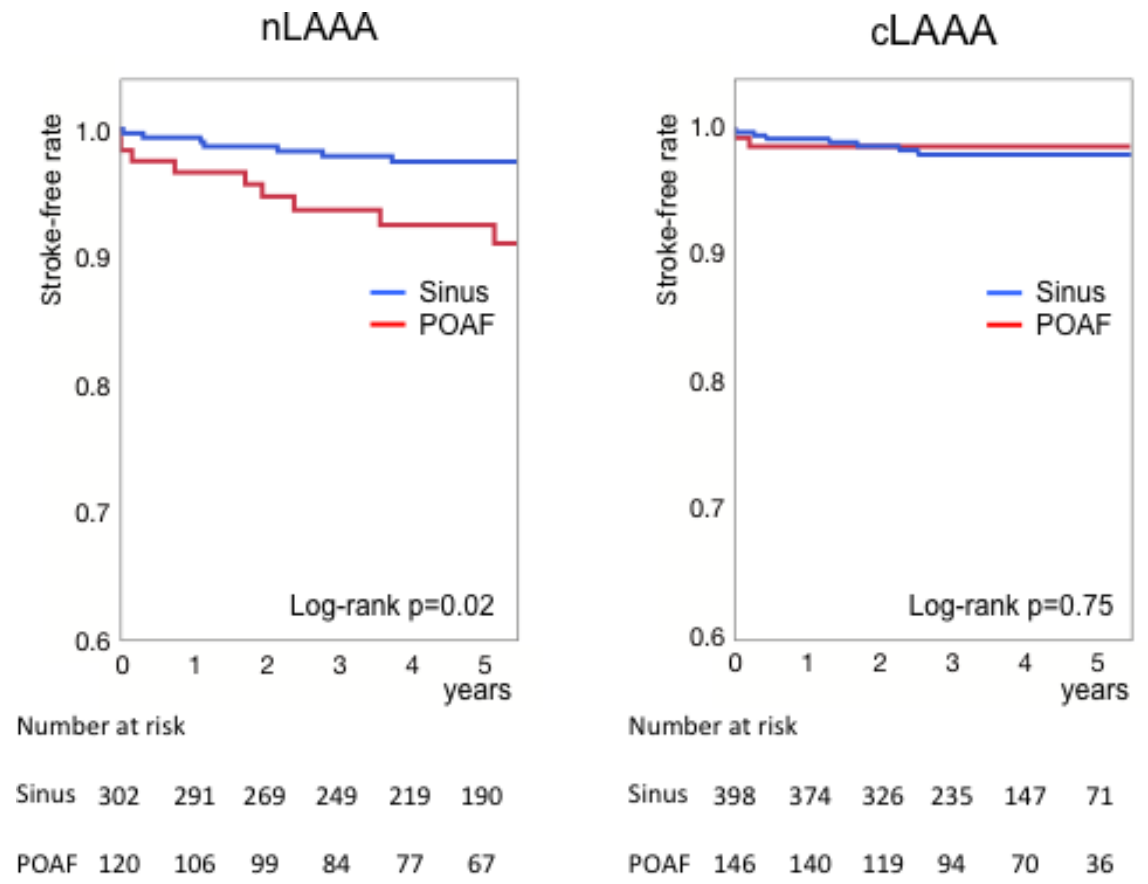

Number at risk

$\begin{array}{lllllll}\text { Sinus } & 398 & 374 & 326 & 235 & 147 & 71\end{array}$

$\begin{array}{lllllll}\text { POAF } & 146 & 140 & 119 & 94 & 70 & 36\end{array}$ 